\title{
Electric-Field-Assisted Growth of Ga-Doped ZnO Nanorods Arrays for Dye-Sensitized Solar Cells
}

\author{
Jinxia Duan', Qiu Xiong1, Jinghua Hu², Hao Wang1* \\ ${ }^{1}$ Hubei Collaborative Innovation Center for Advanced Organic Chemical Materials, Hubei Key Laboratory of \\ Ferro \& Piezoelectric Materials and Devices, Faculty of Physics and Electronic Science, Hubei University, \\ Wuhan, China \\ ${ }^{2}$ School of Science, Wuhan University of Technology, Wuhan, China \\ Email: nanoguy@126.com
}

Received 12 November 2015; accepted 14 December 2015; published 18 December 2015

Copyright (C) 2015 by authors and Scientific Research Publishing Inc.

This work is licensed under the Creative Commons Attribution International License (CC BY).

http://creativecommons.org/licenses/by/4.0/

(c) (i) Open Access

\section{Abstract}

A photoanode with Ga-doped $\mathrm{ZnO}$ nanorods has been prepared on F-doped $\mathrm{SnO}_{2}$ (FTO) coated glass substrate and its application in dye-sensitized solar cells (DSSCs) has been investigated. Gadoped ZnO nanorods have been synthesized by an electric-field-assisted wet chemical approach at $80^{\circ} \mathrm{C}$. Under a direct current electric field, the nanorods predominantly grow on cathodes. The results of the $\mathrm{X}$-ray photoelectron spectroscopy and photoluminescence verify that Ga dopant is successfully incorporated into the ZnO wurtzite lattice structure. Finally, employing Ga-doped ZnO nanorods with the length of $\sim 5 \mu \mathrm{m}$ as the photoanode of DSSCs, an overall energy conversion efficiency of $2.56 \%$ is achieved. The dramatically improved performance of Ga-doped $\mathrm{ZnO}$ based DSSCs compared with that of pure ZnO is due to the higher electron conductivity.

\section{Keywords}

Ga-Doped ZnO, Electric-Field-Assisted, Wet Chemical Method, Dye-Sensitized Solar Cells

\section{Introduction}

Dye-sensitized solar cells (DSSCs) have drawn much attention as a promising renewable energy technology because of their low cost, environmental friendliness and large-scale solar energy conversion [1] [2]. As a result, energy conversion efficiency (PCE) exceeding $12 \%$ has been achieved using mesoporous films of sintered $\mathrm{TiO}_{2}$

${ }^{*}$ Corresponding author.

How to cite this paper: Duan, J.X., Xiong, X., Hu, J.H. and Wang, H. (2015) Electric-Field-Assisted Growth of Ga-Doped ZnO Nanorods Arrays for Dye-Sensitized Solar Cells. Journal of Power and Energy Engineering, 3, 11-18.

http://dx.doi.org/10.4236/jpee.2015.312002 
nanoparticles as the photoanode [3] [4]. The photovoltaic performance of the typical DSSCs with $\mathrm{TiO}_{2}$ nanocrystalline photoanodes has been hampered by electron loss during percolation in the nanoparticle network [5] [6]. However, one-dimensional (1D) nanostructures, such as nanowires (NWs), nanorods (NRs) and nanotubes (NTs), can provide direct electric pathways and reduce the electron recombination, which may improve the performance of photovoltaic devices such as DSSCs [7] [8]. However, most of 1D nanostructured DSSCs have been limited to single-crystalline $\mathrm{ZnO} \mathrm{NRs} / \mathrm{NWs}$ or $\mathrm{TiO}_{2} \mathrm{NWs} / \mathrm{NTs}$ by available synthetic techniques [7]-[12]. Compared to $\mathrm{TiO}_{2}$, $\mathrm{ZnO}$ shows higher electron mobility with similar bandgap and conduction band energies and more abundant morphologies [13]-[15]. Driven by this, $\mathrm{ZnO}$ is regarded as an alternative for high efficient DSSCs. Among the various nanostructures, the application of highly ordered 1D ZnO nanostructures in DSSCs is of prime interest [11] [16].

It is known that nominally undoped $\mathrm{ZnO}$ reveals n-type conduction with a typical carrier concentration of $10^{17} / \mathrm{cm}^{3}$, which is smaller than the carrier concentration of $10^{18}$ to $10^{20} / \mathrm{cm}^{3}$ in optoelectronic applications [17]. The $\mathrm{n}$ - or p-type doping of $\mathrm{ZnO}$ to increase the carrier concentration or decrease the resistivity remains a significant challenge. Controllable n-type doping in $\mathrm{ZnO}$ with high conductivity can be easily achieved by substituting $\mathrm{Zn}$ or O with group III elements such as $\mathrm{Al}, \mathrm{Ga}$, In or group VII elements such as $\mathrm{Cl}, \mathrm{Br}$ and I [18]-[24]. Among these elements, Ga is the most effective n-type dopant in $\mathrm{ZnO}$ since the covalent bond length of Ga-O (1.92 $\AA$ ) is nearly equal to that of $\mathrm{Zn}-\mathrm{O}(1.97 \AA)$. Moreover, the group III elements doped $\mathrm{ZnO}$ are believed to have more potential for diverse applications, including microelectronics, chemical and biological sensor, energy conversion and storage, light-emitting displays, catalysis, drug delivery, and optical storage [18] [19] [23].

Most techniques to obtain $1 \mathrm{D} \mathrm{ZnO}$ require the temperature above $500^{\circ} \mathrm{C}$. The successful growth of $1 \mathrm{D} \mathrm{ZnO}$ via an aqueous solution route suggests an opportunity for low temperature doping [24]-[28]. However, simple addition of group III metal compounds to the growth solution does not result in incorporation of dopants into $\mathrm{ZnO}$ [24]. In this study, we extend the solution method to an electrochemical process by applying a negative potential to the substrate. Ga-doped $\mathrm{ZnO}$ NRs can be obtained at a growth temperature of $80^{\circ} \mathrm{C}$. The optical and electrical properties of Ga-doped ZnO NRs were discussed in detail associated with the results of X-ray photoelectron spectroscopy (XPS) and photoluminescence (PL) measurement. Using 1\% Ga-doped ZnO NRs as photoanode for DSSC, the photovoltaic performance of DSSC was examined. Therefore, this work will help to develop flexible DSSCs and provide a novel strategy of using solar cells for power nanodevices.

\section{Experimental Section}

\subsection{Synthesis of Ga-Doped and Pure ZnO Nanorods}

The synthesis of Ga-doped $\mathrm{ZnO}$ nanorods (NRs) was conducted in an aqueous solution including $9.5 \mathrm{mM}$ $\mathrm{Zn}\left(\mathrm{NO}_{3}\right)_{2} \cdot 6 \mathrm{H}_{2} \mathrm{O}, 0.5 \mathrm{mM} \mathrm{Ga}\left(\mathrm{NO}_{3}\right)_{3} \cdot 6 \mathrm{H}_{2} \mathrm{O}$, and the proper amount of hexamethylenetetramine (HMT). The $\mathrm{pH}$ value of the solution was adjusted to about 7 by addition of appropriate amounts of ammonia. An Electric field was applied between a Zinc sheet anode and a FTO glass substrate cathode in a common two-electrode plating cell at a temperature of around $80^{\circ} \mathrm{C}$ for $1 \mathrm{~h}$. The applied direct current (dc) voltage was $-0.8 \mathrm{~V}$. The distance between the electrodes was $4 \mathrm{~cm}$. After deposition, the samples were washed carefully with deionized water and absolute ethanol before further characterization. For comparison, pure ZnO NRs were grown by the same procedure.

To grow ZnO:Ga nanorod arrays on FTO glass substrates, a ZnO buffered layer was first deposited on the surface of FTO substrates by radio frequency (rf) magnetron sputtering using a $\mathrm{ZnO}$ target with $99.99 \%$ purity at room temperature.

\subsection{Fabrication of Nanorod-Based DSSCs}

Prior to the solar cell assembly, the NRs photoanodes were annealed at $450^{\circ} \mathrm{C}$ for $30 \mathrm{~min}$. Annealed electrodes were soaked in $0.3 \mathrm{mM}$ of ruthenium (II) dye (known as N719, Dyesol) in a tert-butanol/acetonitrile (1:1, V/V) solution for $2 \mathrm{~h}$. The electrodes were washed with acetonitrile, dried and immediately used for assembling the DSSCs. The cells, whose active area was $0.16 \mathrm{~cm}^{2}$, were fabricated by using the dye-adsorbed photoanode and a platinized counter electrode. The two electrodes were clipped together and a Himilan film (Solaronix), with 10 $\mu \mathrm{m}$ thickness, was used as the spacer. The internal space of the cells was filled with liquid electrolyte by capillary action. The electrolyte was composed of $0.5 \mathrm{M}$ butylmethylimidazolium iodide (BMII), $0.05 \mathrm{M}$ iodide $\left(\mathrm{I}_{2}\right)$ 
and 0.5 M 4-tert-butylpyridine and 0.1 M guanidinium thiocyanate (GuNCS) in a solvent mixture of 85\% acetonitrile with $15 \%$ valeronitrile by volume. The DSSCs were then heated to $120^{\circ} \mathrm{C}$ to soften the spacer and seal the edges to prevent the leakage of the electrolyte.

\subsection{Characterization and Measurements}

The size and morphology of as-prepared produces were observed by field-emission scanning electron microscopy (FE-SEM, JSM 6700F). Transmission electron microscope (TEM), high-resolution TEM (HRTEM) images and selected-area electron diffraction (SAED) patterns were obtained on a transmission electron microscope (TEM, FEI Tecnai G2, accelerating voltage: $200 \mathrm{kV}$ ). The chemical compositions of Ga-doped ZnO NRs were measured by X-ray photoelectron spectroscopy (XPS) at room temperature. The photoluminescence (PL) spectra of the samples were characterized using a $325 \mathrm{~nm}$ He-Cd laser. An electrical transport measurement (two-probe) was conducted in a home-built system. A Keithley millimeter was used to determine nanoscale electrical characteristics in nanoscale materials. Photovoltaic measurements were recorded employing an Oriel solar simulator system $\left(100 \mathrm{~mW} / \mathrm{cm}^{2}\right)$ with an AM 1.5 spectrum distribution.

\section{Results and Discussion}

Figure 1 presents (a) plan view and (b) cross-sectional view FE-SEM images of the grown Ga-doped ZnO NRs. It is observed that densely packed arrays of Ga-doped ZnO NRs have been perpendicularly grown on the surfaces of the FTO substrates. The size of the grown NRs is $\sim 100-200 \mathrm{~nm}$ in width and $\sim 5 \mathrm{um}$ in length. The Ga-doped ZnO NRs are uniform, vertically oriented and well-aligned over the entire substrate. The layer appears to be homogeneous and uniform. There is excellent adherence and connection between Ga-doped ZnO NRs and FTO substrate. The morphology of the NRs shows a hexagonal structure, which is due to the wurtzite $\mathrm{ZnO}$ structure.

Transmission electron microscopy (TEM) was employed to characterize the Ga-doped ZnO NRs. The TEM image in Figure 2 depicts the crystal structure of ZnO NRs and the diameter of $100-200 \mathrm{~nm}$. The SAED (the upper inset in Figure 2(a)) demonstrates that the nanorod is a single crystallite. The indexed diffraction spots confirm that it is hexagonal $\mathrm{ZnO}$ with a growth direction along [001] and $\mathrm{Ga}$ has been successfully incorporated
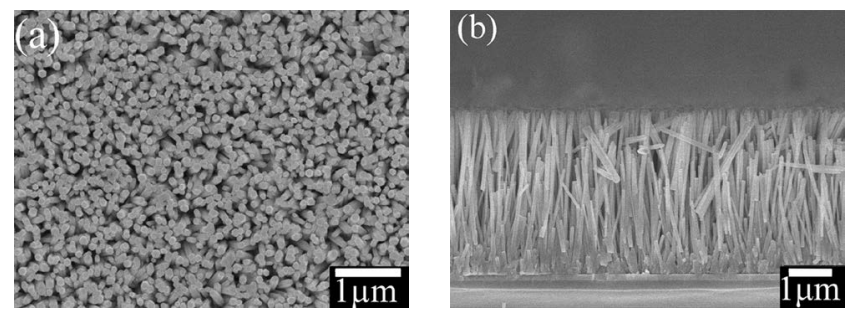

Figure 1. SEM images of Ga-doped ZnO nanorod arrays on FTO glass: (a) plan view; (b) cross-sectional view.

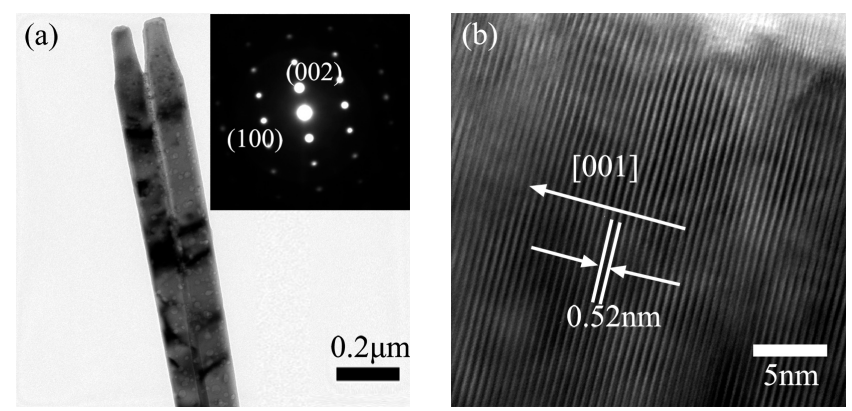

Figure 2. (a) TEM image of Ga-doped $\mathrm{ZnO}$ nanorods, Inset shows selected electron diffraction patterns (SAED); (b) HRTEM image showing individual nanorod with [002] growth direction. Both SAED and HRTEM images are indicative of high crystalline quality for most Ga-doped $\mathrm{ZnO}$. 
into ZnO NRs. The HRTEM image in Figure 2(b) reveals the clear fringes with a spacing of $5.2 \mathrm{~nm}$ corresponding to the (002) plane of hexagonal $\mathrm{ZnO}$. It also demonstrates the [001] direction is the preferential growth direction.

To further confirm the purity and composition of Ga-doped NRs, XPS analysis has been performed. The XPS spectra of Ga 2p and Zn 2p are shown in Figure 3. The spectrum in Figure 3(a) exhibits two significant binding energy peaks at 1117.88 and $1144.35 \mathrm{eV}$ corresponding to the electronic states of Ga 2p3/2 and Ga2p1/2 respectively. Two small satellite peaks come out along with Ga2p peaks, which confirm the successful substitution of Ga into ZnO NRs. Two peaks at 1021.04 and $1044.03 \mathrm{eV}$ corresponding to Zn2p3/2 and Zn2p1/2 appear in Figure 3(b). The energy difference is $26.47 \mathrm{eV}$ between two Ga2p peaks and $22.99 \mathrm{eV}$ between two Zn2p peaks, which agrees with the standard value of 26.84 and $22.97 \mathrm{eV}$, respectively [29] [30]. From close observation, it is learned that binding energy of Ga2p peaks exhibit a positive shift in comparison to standard value of Ga, probably caused by electron transfer from $\mathrm{ZnO}$ to $\mathrm{Ga}$ due to the strong electronic interaction between $\mathrm{Ga}$ and oxide support. In contrast, the binding energy of Zn2p peaks exhibits a negative shift due to the electronegativity $(\chi)$ difference between $\operatorname{Zn}(\chi=1.65)$ and $\mathrm{Ga}(\chi=1.81)$. The scan of O1s spectrum is not shown here, exhibiting a peak at $530.3 \mathrm{eV}$ attributed to oxidized metal ions in the NRs, viz, O-Ga and O-Zn, in the ZnO lattice. This implies that Ga doping can significantly influence the structure of valence band states. Therefore, XPS analysis again confirms the successful incorporation of Ga into ZnO NRs. Samples grown without applied potential were measured no dopant, suggesting the electric field plays a key role in the doping process.

The above electric-field-assisted growth of Ga-doped ZnO NRs was reproducible. The growth behaviors of the NRs were likely attributed to the interaction of electric field and chemical reactivity. When a dc power was applied, the chemical reaction in the solution was adjusted by applied potential, which determined the interfacial free energy. The formation process of ZnO:Ga deposits was investigated here. When the electric field was carried out, $\mathrm{Ga}^{3+}$ and $\mathrm{Zn}^{2+}$ ions will be transferred from bulk solution to cathode, obtaining electrons on the surface of the cathode to form $\mathrm{Zn}$ and Ga atoms (Equations (1)-(2)). These newly formed Ga and $\mathrm{Zn}$ atoms are very active, and rapidly react with $\mathrm{O}_{2}$ and $\mathrm{H}_{2} \mathrm{O}$ in solution to form oxides via reactions. Furthermore, high-temperature $\left(80^{\circ} \mathrm{C}\right)$ can also promote the corrosion of electro-induced $\mathrm{Zn}$ and $\mathrm{Ga}$ atoms to form the stable passive phase $\mathrm{ZnO}$ and $\mathrm{Ga}_{2} \mathrm{O}_{3}$. In addition, the negative potential at the cathode surface resulted in the concentration of hydroxide ions and the consequent formation of $\mathrm{ZnO}$ and $\mathrm{Ga}_{2} \mathrm{O}_{3}$ (Equation (3)-(5)). The produced $\mathrm{ZnO}$ and $\mathrm{Ga}_{2} \mathrm{O}_{3}$ will mix at molecular level, and $\mathrm{Ga}_{2} \mathrm{O}_{3}$ can uniformly enter into the crystal lattices of $\mathrm{ZnO}$, finally leading to the formation of $\mathrm{ZnO}: \mathrm{Ga}^{3+}$ [31].

$$
\begin{aligned}
& \mathrm{Ga}^{3+}+3 \mathrm{e} \rightarrow \mathrm{Ga} \\
& \mathrm{Zn}^{2+}+2 \mathrm{e} \rightarrow \mathrm{Zn} \\
& \mathrm{NO}_{3}^{-}+\mathrm{H}_{2} \mathrm{O}+2 \mathrm{e} \rightarrow \mathrm{NO}_{2}^{-}+2 \mathrm{OH}^{-} \\
& \mathrm{Zn}^{2+}+2 \mathrm{OH}^{-} \rightarrow \mathrm{ZnO}+\mathrm{H}_{2} \mathrm{O} \\
& \mathrm{Ga}^{3+}+3 \mathrm{OH}^{-} \rightarrow \mathrm{Ga}_{2} \mathrm{O}_{3}+\mathrm{H}_{2} \mathrm{O}
\end{aligned}
$$

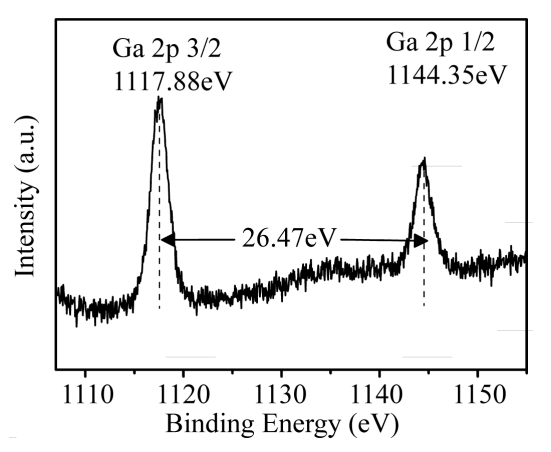

(a)

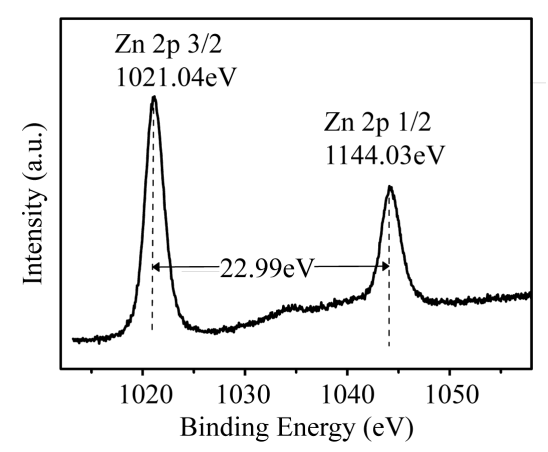

(b)

Figure 3. XPS spectra of Ga-doped ZnO nanorods corresponding to (a) Ga2p and (b) Zn2p. 
Room-temperature PL curves in Figure 4(a) reveal that the near-band edge (NBE) emission peak shifts from $376.3 \mathrm{~nm}(3.29 \mathrm{eV})$ in pure $\mathrm{ZnO}$ to $382.5 \mathrm{~nm}$ (3.25 eV) in Ga-doped ZnO, while the emission intensity decreases with Ga doping. A small red shift in UV emission and the apparent band gap narrowing related to Ga doping are attributed to semiconductor-to-metal transition [18] [32] [33]. The band gap of a semiconductor increases when the impurity concentration is below Mott's critical density. However, an abrupt decrease in band gap would occur when the impurity concentration is above Mott's critical density. In the present study, the red shifts in the emission peak of Ga-doped ZnO NRs $(6.2 \mathrm{~nm})$ suggest that the impurity concentration is higher than Mott's critical density. Actually, Ga heavily doped ZnO shows metallic behavior [18]. The intensity of NBE emission decreases slightly in the Ga-doped sample as compared to the pure one, and all the PL spectra are normalized to maximum emission peaks. A significant difference is observed in the intensity ratio of NBE emission to the defect emission centered at $\sim 580 \mathrm{~nm}$, which is usually ascribed to the O-related native defect states in ZnO. In fact, such defects are clearly suppressed in the Ga-doped sample. One should note that Ga doping in $\mathrm{ZnO}$ modify the electronic structure of ZnO. At low doping concentrations (below $10^{20} / \mathrm{cm}^{3}$ ), the substitutional Ga atoms on $\mathrm{Zn}$ sites form a shallow donor level, which will increase the free electron density, compensate the positive $\mathrm{O}$ vacancy defects, and suppress $\mathrm{O}$ vacancy formation in the $\mathrm{ZnO}$ lattice [34]. This is consistent with our experimental observation of depressed $\mathrm{O}$ defect emission in room-temperature PL spectrum of the low doping concentration of Ga.

The schematic illustration of NRs/buffer-layer/FTO glass substrate and its external circuit for I-V measurement are shown in Figure 4(b). For ohmic contact, an Au/Ti bilayer was deposited by magnetron sputtering on the surface of Ga-doped ZnO NRs and FTO substrate, and patterned with a shadow mask. I-V spectra on undoped $\mathrm{ZnO}$ and Ga-doped ZnO NRs as well as calibration experiments were performed to ensure reproducibility. Black curves and red curves correspond to the spectra of undoped and Ga-doped ZnO NRs, respectively. A significantly steeper rising slope in I-V spectra is clear in Ga-doped ZnO NRs in comparison with those in the undoped NRs. It confirms the conductivity enhancement in NRs due to Ga doping, which is similar to the reports [32] [35].

In a preliminary attempt, Ga-ZnO NRs with a thickness of approximately $5 \mu \mathrm{m}$ were fabricated for use as photoanode in DSSC tests (see Figure 5). For comparison, an undoped ZnO NRs photoanode with the same thickness was also constructed. Figure 6 demonstrates the characteristic photocurrent-voltage (J-V) curves under AM 1.5 sunlight illumination $\left(100 \mathrm{~mW} \cdot \mathrm{cm}^{-2}\right)$ and their photovoltaic parameters derived from the $\mathrm{J}-\mathrm{V}$ curves are summarized in Table 1 . An overall solar to electrical PCE $(\eta)$ of $2.56 \%$ was achieved for Ga-ZnO NRs based DSSC with a current density (Jsc) of $8.88 \mathrm{~mA} \cdot \mathrm{cm}^{-2}$, opencircuit voltage $\left(\mathrm{V}_{\mathrm{OC}}\right)$ of $0.59 \mathrm{~V}$, and fill factor (FF) of 0.49 , much higher than the performance of ZnO NRs based DSSC $\left(\eta=1.07 \%\right.$, JsC $=4.50 \mathrm{~mA} \cdot \mathrm{cm}^{-2}, \mathrm{~V}_{\mathrm{OC}}$ $=0.55 \mathrm{~V}, \mathrm{FF}=0.43)$. The data demonstrates that PCE can be enhanced more than 2 times by doping Ga into ZnO. The improvement in PCE value of Ga-ZnO NRs DSSC is obviously caused by the higher Jsc. The significantly enhanced PCE and Jsc in Ga-doped ZnO based DSSCs could be attributed to the improved conductivity

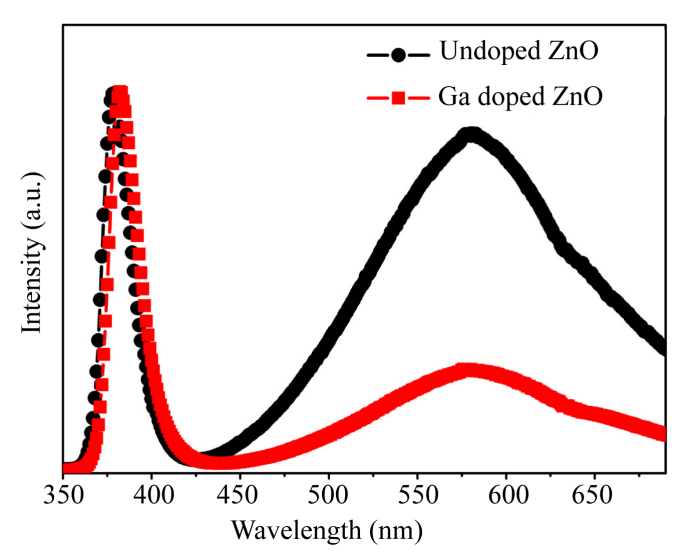

(a)

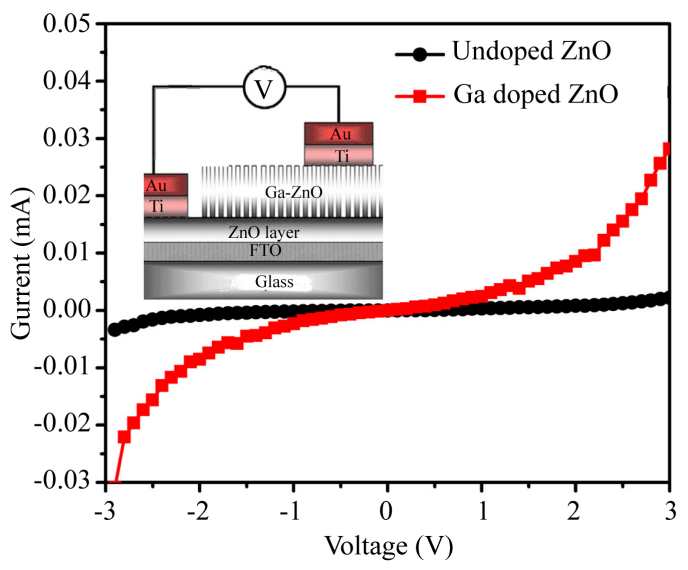

(b)

Figure 4. (a) Room-temperature PL spectra of undoped and Ga-doped ZnO NRs. (b) I-V characteristics of undoped and Ga-doped ZnO nanorods. The inset shows the schematic illustration of the nanorods/buffer-layer/FTO glass and its external circuit for I-V measurement. 


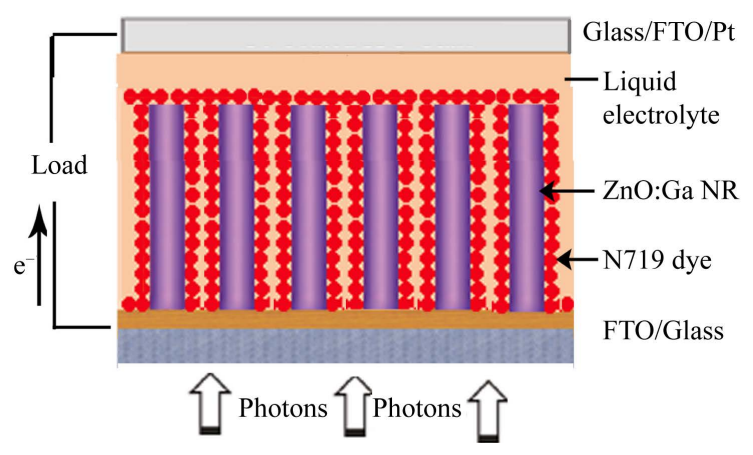

Figure 5. Schematic illustration of the configuration of a dye sensitized Ga-doped ZnO nanorods (ZnO:Ga NRs) Solar Cell.

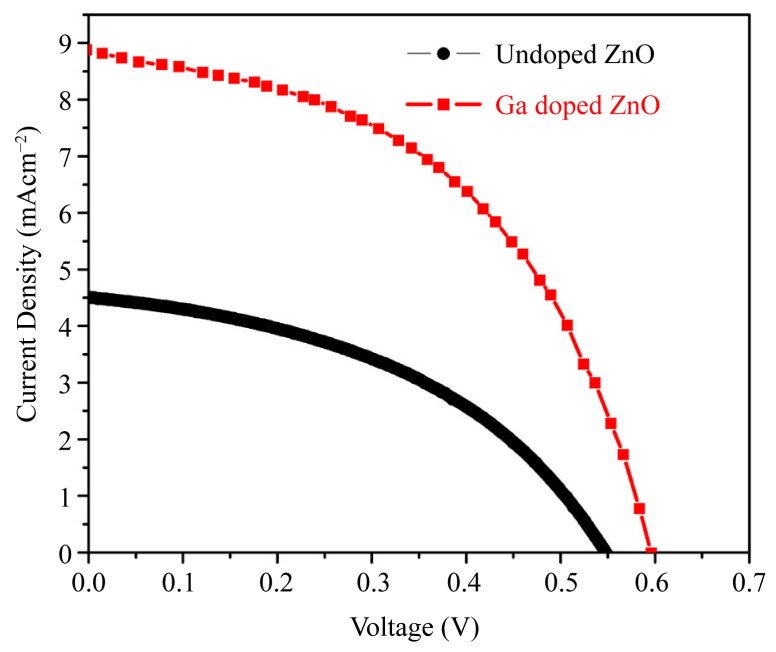

Figure 6. J-V characteristics (full line) for $\mathrm{ZnO}$ nanowire arrays electrodeposited on FTO electrode $\left(0.25 \mathrm{~cm}^{2}\right)$ sensitized with N719 (simulated AM1.5G illumination with intensity $100 \mathrm{~mW} / \mathrm{cm}^{2}$ ).

Table 1. Photovoltaic parameters of the Ga-ZnO NRs and ZnO NR based DSCs under AM 1.5 sunlight illumination (100 $\left.\mathrm{mW} \cdot \mathrm{cm}^{-2}\right)$.

\begin{tabular}{ccccc}
\hline Sample & $\mathrm{J}_{\mathrm{sc}}\left(\mathrm{mA} \cdot \mathrm{cm}^{-2}\right)$ & $\mathrm{V}_{\mathrm{oc}}(\mathrm{V})$ & $\mathrm{FF}$ & Efficiency $(\%)$ \\
\hline $\mathrm{Ga}-\mathrm{ZnO}$ & 8.88 & 0.59 & 0.49 & 2.56 \\
$\mathrm{ZnO}$ & 4.50 & 0.55 & 0.43 & 1.07 \\
\hline
\end{tabular}

of Ga-doped ZnO layer. The slightly higher $\mathrm{V}_{\mathrm{OC}}$ of Ga-ZnO based DSSCs should be a result of the addition of Ga into the $\mathrm{ZnO}$ to decrease charge recombination [36].

The thickness of $\mathrm{ZnO}$ thin films in our device is about $5 \mu \mathrm{m}$, much thinner than that in the previous work, resulting in a decrease of PCE due to deterioration of the transparency [11] [37]. We can further improve the PCE of our DSSCs with either pure $\mathrm{ZnO}$ or $\mathrm{Ga}$-doped $\mathrm{ZnO}$ by controlling thicknesses of a nanostructured $\mathrm{ZnO}$ layer. We believe that the current PCE (2.56\% for Ga-doped ZnO based DSSCs and 1.07\% for pure ZnO based DSSCs) is enough to evaluate Ga-doping effect on the active layer in DSSCs.

\section{Conclusion}

Ga-doped ZnO NRs were successfully prepared on FTO substrates by the electric-field-assisted wet chemical method. XPS and PL results manifested that Ga atoms had been doped effectively into the ZnO lattices. A $5 \mu \mathrm{m}$ thick Ga-doped ZnO NRs photoanode based DSSC showed a solar to electrical energy conversion efficiency 
(PCE) of 2.56\%, while the efficiency of a cell with ZnO NRs photoanode was $1.07 \%$. The dramatic enhancement of the PCE of the Ga-doped ZnO-based DSSCs is due to the higher electron conductivity. Further experiments are underway to investigate the effect of the fundamental geometrical features of Ga-ZnO NRs on solar cell conversion efficiency. These will lead to new strategies for improvement.

\section{Acknowledgements}

This work was partially supported by the National Nature Science Foundation of China (Nos. 51072049 and 11204070), ED of Hubei Province (Nos. 2009CDA035, 2010BFA016, Z20091001 and Q20120106).

\section{References}

[1] O’Regan, B. and Grätzel, M. (1991) A Low-Cost, High-Efficiency Solar Cell Based on Dye-Sensitized Colloidal TiO 2 Films. Nature, 353, 737-740. http://dx.doi.org/10.1038/353737a0

[2] Chen, C.Y., Wang, M.K., Li, J.Y., Pootrakulchote, N., Alibabaei, L., Ngoc-le, C., Decoppet, J., Tsai, J., Grätzel, C., Wu, C., Zakeeruddin, S.M. and Grätze, M. (2009) Highly Efficient Light-Harvesting Ruthenium Sensitizer for ThinFilm Dye-Sensitized Solar Cells. ACS Nano, 3, 3103-3109. http://dx.doi.org/10.1021/nn900756s

[3] Yella, A., Lee, H.W., Tsao, H.N., Yi, C.Y., Chandiran, A.K., Nazeeruddin, M.K., Diau, E.W.G., Yeh, C.Y., Zakeeruddin, S.M. and Grätzel, M. (2011) Porphyrin-Sensitized Solar Cells with Cobalt (II/III)—Based Redox Electrolyte Exceed 12 Percent Efficiency. Science, 334, 629-634. http://dx.doi.org/10.1126/science.1209688

[4] Hagfeldt, A. (2012) Brief Overview of Dye-Sensitized Solar Cells. Ambio, 41, 151-155.

[5] Bazzan, G., Deneault, J.R., Kang, T., Taylor, B.E. and Durstock, M.F. (2011) Nanoparticle/Dye Interface Optimization in Dye-Sensitized Solar Cells. Advanced Functional Materials, 21, 3268-3274. http://dx.doi.org/10.1002/adfm.201100595

[6] Tétreault, N., Arsenault, É., Heiniger, L.P., Soheilnia, N., Brillet, J., Moehl, T., Zakeeruddin, S., Ozin, G.A. and Grätzel, M. (2011) High-Efficiency Dye-Sensitized Solar Cell with Three-Dimensional Photoanode. Nano Letters, 11, 4579-4584. http://dx.doi.org/10.1021/nl201792r

[7] Desai, U.V., Xu, C.K., Wu, J.M. and Gao, D. (2013) Hybrid $\mathrm{TiO}_{2}-\mathrm{SnO}_{2}$ Nanotube Arrays for Dye-Sensitized Solar Cells. Journal of Physical Chemistry C, 117, 3232-3239. http://dx.doi.org/10.1021/jp3096727

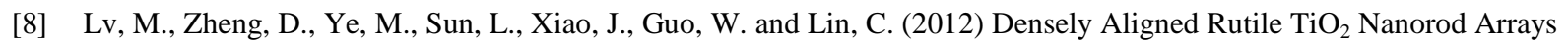
with High Surface Area for Efficient Dye-Sensitized Solar Cells. Nanoscale, 4, 5872-5879. http://dx.doi.org/10.1039/c2nr31431b

[9] Irene, G.V. and Monica, L.C. (2009) Vertically-Aligned Nanostructures of ZnO for Excitonic Solar Cells: A Review. Energy \& Environmental Science, 2, 19-34. http://dx.doi.org/10.1039/B811536B

[10] Wang, X.N., Zhu, H.J., Xu, Y.M., Wang, H., Tao, Y., Hark, S., Xiao, X.D. and Li, Q. (2010) Aligned ZnO/CdTe CoreShell Nanocable Arrays on Indium Tin Oxide. ACS Nano, 4, 3302-3308. http://dx.doi.org/10.1021/nn1001547

[11] Xu, C.K., Wu, J.M., Desai, U.V. and Gao, D. (2011) Multilayer Assembly of Nanowire Arrays for Dye-Sensitized Solar Cells. Journal of the American Chemical Society, 133, 8122-8125. http://dx.doi.org/10.1021/ja202135n

[12] Wang, H., Wang, T., Wang, X.N., Liu, R., Wang, H.B., Xu, Y., Zhang, J. and Duan, J.X. (2012) Double-Shelled ZnO/ CdSe/CdTe Nanocable Arrays for Photovoltaic Applications: Microstructure Evolution and Interfacial Energy Alignment. Journal of Materials Chemistry, 22, 12532-12537. http://dx.doi.org/10.1039/c2jm32253f

[13] Son, D.Y., Im, J.H., Kim, H.S. and Park, N.G. (2014) 11\% Efficient Perovskite Solar Cell Based on ZnO Nanorods: An Effective Charge Collection System. Journal of Physical Chemistry C, 118, 16567-16573. http://dx.doi.org/10.1021/jp412407j

[14] Duan, J.X., Huang, X.T., Wang, E.K. and Ai, H.H. (2006) Synthesis of Hollow ZnO Microspheres by an Integrated Autoclave and Pyrolysis Process. Nanotechnology, 17, 1786-1790. http://dx.doi.org/10.1088/0957-4484/17/6/040

[15] Zeng, H.B., Duan, G.T., Li, Y., Yang, S.K., Xu, X.X. and Cai, W.P. (2010) Blue Luminescence of ZnO Nanoparticles Based on Non-Equilibrium Processes: Defect Origins and Emission Controls. Advanced Functional Materials, 20, 561572. http://dx.doi.org/10.1002/adfm.200901884

[16] Law, M., Greene, L.E., Johnson, J.C., Saykally, R. and Yang, P.D. (2005) Nanowire Dye-Sensitized Solar Cells. Nature Materials, 4, 455-459. http://dx.doi.org/10.1038/nmat1387

[17] Xu, C.K., Chun, J.W., Kim, D.E., Kim, J., Chon, B. and Joo, T. (2007) Electrical Properties and Near Band Edge Emission of Bi-Doped ZnO Nanowires. Applied Physics Letters, 90, Article ID: 083113. http://dx.doi.org/10.1063/1.2431715 
[18] Yao, Y.F., Tu, C.G., Chang, T.W., Chen, H.T., Weng, C.M., Su, C.Y., Hsieh, C., Liao, C.H., Kiang, Y.W. and Yang, C.C. (2015) Growth of Highly Conductive Ga-Doped ZnO Nanoneedles. ACS Applied Materials \& Interfaces, 7, 10525-10533. http://dx.doi.org/10.1021/acsami.5b02063

[19] Ahmad, M., Sun, H. and Zhu, J. (2011) Enhanced Photoluminescence and Field-Emission Behavior of Vertically Well Aligned Arrays of In-Doped ZnO Nanowires. ACS Applied Materials \& Interfaces, 3, 1299-1305. http://dx.doi.org/10.1021/am200099c

[20] Yuan, G.D., Zhang, W.J., Jie, J.S., Fan, X., Tang, J.X., Shafiq, I., Ye, Z.Z., Lee, C.S. and Lee, S.T. (2008) Tunable $n$ Type Conductivity and Transport Properties of Ga-Doped ZnO Nanowire Arrays. Advanced Materials, 20, 168-173. http://dx.doi.org/10.1002/adma.200701377

[21] Onwona-Agyeman, B., Nakao, M., Kohno, T., Liyanage, D., Murakam, K.I. and Kitaoka, T. (2013) Preparation and Characterization of Sputtered Aluminum and Gallium Co-Doped ZnO Films as Conductive Substrates in Dye-Sensitized Solar Cells. Chemical Engineering Journal, 219, 273-277. http://dx.doi.org/10.1016/j.cej.2013.01.006

[22] Du, S.F., Liu, H. and Chen, Y. (2009) Large-Scale Preparation of Porous Ultrathin Ga-Doped ZnO Nanoneedles from 3D Basic Zinc Carbonate Superstructures. Nanotechnology, 20, Article ID: 085611. http://dx.doi.org/10.1088/0957-4484/20/8/085611

[23] Yoo, J., Lee, C., Joo Doh, Y.H., Jung, S. and Yi, G.C. (2009) Modulation Doping in ZnO Nanorods for Electrical Nanodevice Applications. Applied Physics Letters, 94, Article ID: 223117. http://dx.doi.org/10.1063/1.3148666

[24] Wang, H., Baek, S., Song, J., Lee, J. and Lim, S. (2008) Microstructural and Optical Characteristics of Solution-Grown Ga-Doped ZnO Nanorod Arrays. Nanotechnology, 19, Article ID: 075607. http://dx.doi.org/10.1088/0957-4484/19/7/075607

[25] Wang, H., Wang, H.B., Yang, F.J., Chen, Y., Zhang, C., Yang, C.P., Qi, L. and Wong, S.P. (2006) Structure and Magnetic Properties of $\mathrm{Zn}_{1-x} \mathrm{Co}_{x} \mathrm{O}$ Single-Crystalline Nanorods Synthesized by a Wet Chemical Method. Nanotechnology, 17, 4312-4316. http://dx.doi.org/10.1088/0957-4484/17/17/005

[26] Duan, J.X., Wang, H., Wang, H.B., Zhang, J., Wu, S. and Wang, Y. (2012) Mn-Doped ZnO Nanotubes: From Facile Solution Synthesis to Room Temperature Ferromagnetism. CrystEngComm, 14, 1330-1336. http://dx.doi.org/10.1039/C1CE06221B

[27] Wang, H., Chen, Y., Wang, H.B., Zhang, C., Yang, F.J., Duan, J.X., Yang, C.P., Xu, Y.M., Zhou, M.J. and Li, Q. (2007) High Resolution Transmission Electron Microscopy and Raman Scattering Studies of Room Temperature Ferromagnetic Ni-Doped ZnO Nanocrystal. Applied Physics Letters, 90, Article ID: 052505.

[28] Zhou, H., Fang, G.J., Liu, N. and Zhao, X.Z. (2011) Effects of Thermal Annealing on the Performance of Al/ZnO Nanorods/Pt Structure Ultraviolet Photodetector. Materials Science and Engineering B, 176, 740-744. http://dx.doi.org/10.1016/j.mseb.2011.03.003

[29] Wagner, C.D., Riggs, W.M., Davis, L.E., Monlder, J.I. and Muilenberg, G. E. (1979) In Handbook of X-Ray Photoelectron Spectroscopy. Perkin-Elmer Corporation, Eden Prarie, 171-174.

[30] Bae, S.Y., Na, C.W., Kang, J.H. and Park, J. (2005) Comparative Structure and Optical Properties of Ga-, In-, and SnDoped ZnO Nanowires, Synthesized via Thermal Evaporation. The Journal of Physical Chemistry B, 109, $2526-2531$. http://dx.doi.org/10.1021/jp0458708

[31] Li, G.R., Lu, X.H., Su, C.Y. and Tong, Y.X. (2008) Low-Temperature Growth and Characterization of Cl-Doped ZnO Nanowire Arrays. Journal of Physical Chemistry, 112, 2927-2933.

[32] Zhou, M.J., Zhu, J.H., Jiao, Y., Rao, Y.Y., Hark, S., Liu, Y., Peng, L.M. and Li, Q. (2009) Optical and Electrical Properties of Ga-Doped ZnO Nanowire Arrays on Conducting Substrates. Journal of Physical Chemistry, 113, 8945-8947.

[33] Mott, N.F. (1974) Metal-Insulator Transitions. Taylor and Francis, London.

[34] Matsui, H., Saeki, H., Tabata, H. and Kawai, T. (2003) Role of Ga for Co-Doping of Ga with N in ZnO Films. Japanese Journal of Applied Physics, 42, 5494-5499. http://dx.doi.org/10.1143/JJAP.42.5494

[35] Yang, P.Y., Wang, H., Wang, X.N., Zhang, J. and Jiang, Y. (2010) Optical and Electrical Properties of Ga-Doped ZnO Nanorod Arrays Fabricated by Catalyst-Free Thermal Evaporation. Proceedings of the 3rd International Nanoelectronics Conference, Hong Kong, 3-8 January 2010, 1187-1188. http://dx.doi.org/10.1109/inec.2010.5424956

[36] Shin, K.S., Lee, K.H., Lee, H.H., Choi, D. and Kim, S.W. (2010) Enhanced Power Conversion Efficiency of Inverted Organic Solar Cells with a Ga-Doped ZnO Nanostructured Thin Film Prepared Using Aqueous Solution. Journal of Physical Chemistry C, 114, 15782-15785. http://dx.doi.org/10.1021/jp1013658

[37] Zhang, Q.F., Dandeneau, C.S., Zhou, X.Y. and Cao, G.Z. (2009) ZnO Nanostructures for Dye-Sensitized Solar Cells. Advanced Materials, 21, 4087-4108. http://dx.doi.org/10.1002/adma.200803827 